\title{
Unconventional vesicle-to-ribbon transition behaviour of diacyl glycerol amino acid based surfactants in extremely diluted systems induced by pH-concentration effects $\dagger$
}

\author{
Aurora Pinazo, Lourdes Pérez, María Rosa Infante and Ramon Pons* \\ Departament de Tecnologia de Tensioactius, Institut d'Investigacions Químiques i Ambientals de \\ Barcelona, CSIC, Jordi Girona 18-26, 08034 Barcelona, Spain. E-mail: rppten@iiqab.csic.es
}

Received 21st October 2003, Accepted 9th February 2004

First published as an Advance Article on the web 1st March 2004

\begin{abstract}
A vesicle-to-ribbon transition has been observed in extremely dilute 1- $O$-(L-arginyl)-2, 3-O-di-decanoyl-snglycerol dichlorohydrate (1010R) surfactant systems by means of static light scattering. At concentration as low as $0.005 \mathrm{mM}$ the solutions scattered significantly. From the angular dependence and molecular weight of the aggregates a vesicular structure is suggested. Increasing surfactant concentration in water induces the vesicle-toribbon transition at concentrations as low as $0.5 \mathrm{mM}$. This transition is accompanied by a strong decrease of scattered intensity and change in angular dependence. Lowering the $\mathrm{pH}$ at a fixed concentration can induce the same transition. Both parameters change the protonation of the surfactant, inducing an increase in preferred surfactant head-group area. Those findings are congruent with the observed surface tension behaviour as a function of surfactant concentration and suggests an explanation for the widely different critical micellar concentration (c.m.c.) as determined by different techniques.
\end{abstract}

\section{Introduction}

Manufacturers and consumers have an increasing interest for novel environmentally friendly surfactants; naturally occurring amino acids have been of particular interest in this field. Surfactant molecules from renewable raw materials that mimic natural lipo-amino acids are one of the preferred choices for food, pharmaceutical, and cosmetic applications. A series of novel cationic arginine amino acid-based surfactants has been synthesized. ${ }^{1,2}$ Theses surfactants exhibit considerable antimicrobial activity, satisfactory toxicity profiles and high biodegradability.

The behaviour of monomeric and gemini arginine-based surfactants have been extensively studied; monomeric surfactants show one critical micelle concentration (c.m.c.) and forms "normal" micelles. Gemini surfactants arginine-based show unconventional aggregation behaviour and two c.m.c.s were inferred from several techniques. ${ }^{3}$

A new glycerol-arginine-based double-chain surfactant 1010R (Fig. 1) has been synthesized. ${ }^{4,5}$ This new surfactant have two aliphatic chains and the arginine amino acid as polar head group linked together through a glycerol moiety. It can be considered analogue to a partial glyceride and phospholipid, exhibiting the properties of both glycerides and lecithins, along with and improved water solubility.

Due to their amphiphyllic character, surfactants form in solution a variety of structures. Increasing concentration, the usual sequence of structures formed by surfactants in water is; monomers-spherical-rod-lamellae-inverse rod-inverse sphere. ${ }^{6}$ This sequence is governed by changes in the ratio of the preferred surfactant polar head area to the hydrophobic volume. ${ }^{7-10}$ Increasing concentration leads to a decrease in area per molecule induced by ionic strength producing cylinder to disk transitions. ${ }^{11}$ Transitions from cylinders to vesicle were observed for lecithin-bile salt mixed systems when reducing the bile salt contents. ${ }^{12}$ These type of changes can also be induced

$\dagger$ Presented at the 17th Conference of the European Colloid \& Interface Science Society, Firenze, Italy, September 21-26, 2003. at constant surfactant concentration by increasing the ionic strength of the water medium, ${ }^{13-15}$ by the presence of cosurfactants in the system ${ }^{16}$ or also by changing temperature. ${ }^{11}$

The critical micellar concentration corresponds to the transition between single dispersed surfactant molecules to spherical aggregates. This critical concentration can be detected by several techniques such as surface tension, conductivity, light scattering etc. The ability of these techniques to detect this transition depends on the physical quantity measured. For instance, the detection of the c.m.c. by surface tension relays on the difference in adsorption of monomers and micelles at the surface, once micelles start forming, the activity of monomers changes little and the amount of monomer adsorbed remains near constant. Conductivity detects the c.m.c. by changes of the relative conductivity of monomers and micelles which are less conducting. Light scattering detects the c.m.c. by the difference in scattering power of the monomers and micelles that scatter more due to their bigger size. Slightly different values for c.m.c. are usually encountered by using different techniques because they probe different phenomena. ${ }^{3}$

In gemini surfactants two critical concentrations have been encountered, ${ }^{3,17-19}$ these two critical concentrations have been

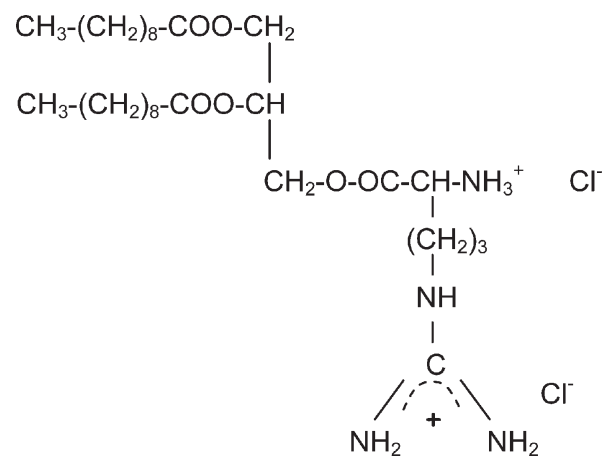

Fig. 1 Molecular structure of the 1-O-(L-arginyl)-2,3-O-di-dodecanoyl-sn-glycerol dichlorhydrate (1010R) surfactant. 
attributed to a true c.m.c. and to the formation of pre-micellar aggregates or ion pairing at lower concentration.

Phospholipids and mimetic molecules have very small monomer solubility and usually no c.m.c. is detected. ${ }^{20}$ These types of molecules form lamellar structures which could be open or closed to form vesicles. In this kind of systems several studies have detected different vesicles to micelle transitions. This transition is usually induced by addition of a solubilising surfactant. ${ }^{21}$ The contrary effect, micelle-to-vesicle, transition upon dilution has also been shown to occur in those mixed systems because the partitioning of the most soluble molecule in the aggregates changes with concentration and, at low concentration the aggregates are enriched in the vesicle forming molecule. $^{22}$

Similarly to gemini surfactants, unconventional aggregation behaviour and two c.m.c.s were inferred from surface tension, conductivity and fluorescence for the new 1010R surfactant. The first c.m.c., or c.m.c.1 was detected by surface tension measurements. Fluorescence and conductivity data indicate clearly a conventional c.m.c. 2 at about an order of magnitude higher than that from the tension results. In this article the influence of the two cationic groups on the aggregation behaviour is presented and discussed. In this article we show for the first time a vesicle-to-ribbon transition induced by increasing surfactant concentration. We discuss the possible influence of the charge of the polar head on this behaviour.

\section{Static light scattering fundamentals}

Static light scattering is a useful technique for the determination of aggregation and aggregate structure in dilute systems. In dilute systems the aggregates can be considered independent scatterers and the total scattered intensity is simply the sum of the scattering intensity of the individual aggregates. In the limit of low concentration the Rayleigh ratio $\left(R_{\theta}\right)$ at the measured angle $\theta$ is:

$$
R_{\theta}=\frac{I_{\theta}-I_{\theta}^{0}}{R_{\theta}^{s}}
$$

and the relation between $R_{\theta}$ and the optical constant $(K)$ is:

$$
\frac{K c}{R_{\theta}}=\frac{1}{M P(q, r)}
$$

where $c$ is the concentration of aggregated material, $M$ the molar mass of the aggregates, $P(q, r)$ is the intra-particle structure factor (see below) or form factor.

The optical constant value corresponds to:

$$
K=\frac{(2 \pi n(\mathrm{~d} n / \mathrm{d} c))^{2}}{N_{\mathrm{A}} \lambda^{4}}
$$

where $n$ is the refractive index of the solution, $\mathrm{d} n / \mathrm{d} c$ is the refractive index increment, $N_{\mathrm{A}}$ Avogadro's number and $\lambda$ the wavelength of the incident light.

The form factor $P(q, r)$ contains the angular dependence of scattered light for an object which form is defined by the generalized coordinates $\boldsymbol{r}$. The variable $q$, scattering vector modulus, corresponds to eqn. (4):

$$
q=\frac{4 \pi}{\lambda} \sin (\theta / 2)
$$

In the Rayleigh-Debye-Gans (RDG) limit, $2 \pi L / \lambda(m-1) \ll$ $1,{ }^{23}$ where $m$ is the ratio of particle refractive index over medium refractive index and $L$ the maximum size of the particle that is; small aggregates of material with refractive index close to the medium, the form factor can be explicitly calculated. ${ }^{24,25}$

In the case of vesicles the refractive index of the object is quite close to the medium and this approximation can be applied. Shell models are constructed by subtraction of a compact model with a smaller model with the same properties. Arbitrary shapes can be built by Fourier transformation of the pair distribution function ${ }^{26}$

For very small particles $(L \ll \lambda)$ at small angles the form factor is close to unity, increasing length leads to angular dependence in the scattering. In the Guinier approximation the scattering of globular particles can be approximated as eqn. (5):

$$
I=I_{0} \exp \left(-\frac{\left(R_{\mathrm{g}} q\right)^{2}}{3}\right)
$$

this approximation is valid for $R_{\mathrm{g}} q<1$, however, this approximation usually works within a few percent even for $R_{\mathrm{g}} q$ above unity. $^{27}$

A $\log (I)$ versus $q^{2}$ plot would be linear for globular geometry. For cylindrical geometry the generalized Guinier behaviour corresponds to eqn. (6):

$$
I q=I_{0} \exp \left(-\frac{\left(R_{\mathrm{g}} q\right)^{2}}{2}\right)
$$

and a plot of $\log (I q)$ versus $q^{2}$ would show linear behaviour; finally for flat particles we have the generalized Guinier approximation as eqn. (7):

$$
I q^{2}=I_{0} \exp \left(-\frac{\left(R_{\mathrm{g}} q\right)^{2}}{1}\right)
$$

A $\log \left(I q^{2}\right)$ versus $q^{2}$ plot of a locally flat particle would correspond to a linear decrease.

We should also bear in mind that any intensity behaviour could be represented by a polydisperse population. If a population of spheres with an adequate size distribution is present, the global intensity behaviour could resemble that of a cylinder population or lamellae. This uncertainty between shape and polydispersity is always present in scattering experiments. Up to a certain point, the absolute intensity scaling could favour one or other type of interpretations. By combining absolute intensity with angular dependence the size and wall thickness of vesicles can be obtained, ${ }^{28}$ in this case the fit of the angular dependence mainly influences the radius of the vesicle and the absolute scaling mainly influences the wall thickness.

\section{Materials and methods}

\subsection{Materials}

The 1010R surfactant was synthesized with the method described in ref. 4 . The product purity was examined by high performance liquid chromatography, ${ }^{1} \mathrm{H}-\mathrm{NMR},{ }^{13} \mathrm{C}-\mathrm{NMR}$, mass spectroscopy and elemental analysis. The results shown that purity was higher than $99 \%$.

Millipore water from a Milli-Q four-bowl system was used in preparing all sample solutions; the water used for light scattering measurements was also filtered through $0.2 \mu \mathrm{m}$ Nucleopore membrane.

The hydrochloric acid used for acidification of a sample was Merck 25\% aqueous solution.

\section{Methods}

Static light scattering. The samples for light scattering were prepared by dilution from a concentrated sample of $1.5 \mathrm{mM}$ prepared by direct weighing of the surfactant and water. The water was previously filtered through $0.2 \mu \mathrm{m}$ filters from Osmonics Inc. Static light scattering was performed with a Malvern 4700c PCS system. Quartz cylindrical cells were used; some samples were measured with $1 \mathrm{~cm}$ diameter cells and 
some with $4 \mathrm{~cm}$ diameter cells. No differences were found except for increased scattering at the angle of $30^{\circ}$ in the case of $1 \mathrm{~cm}$ diameter cells, also indicating the absence of appreciable multiple scattering. $1 \mathrm{~mm}$ aperture was used in the photo multiplier to assure a high enough intensity. An argon laser Cyonics, model 2213-73SLYV, Malvern 4700c with a $488 \mathrm{~nm}$ vertically polarized operated in the intensity mode was used as light source. Absolute intensity was obtained from the comparison of the intensity with a toluene sample. A water sample was subtracted of all the samples. Both toluene and water samples produced scattered intensities which, after angular correction, gave constant intensity except for the above-mentioned angle of $30^{\circ}$ with $1 \mathrm{~cm}$ diameter cells; this assures the correct alignment of our equipment. The intensity was measured for $250 \mathrm{~s}$ in $1 \mathrm{~s}$ intervals. The mean of the lower 25 measurements was taken as the measured intensity. This procedure allows us to discard part of the signal produced by dust or other impurities that would increase the intensity of scattered light. No significant increase of the intensity was observed even taking the mean of the lowest 200 values, only the dispersion increased typically to $0.5 \%$. The scattering intensity was measured at $15^{\circ}$ intervals from $30^{\circ}$ to $120^{\circ}$.

Surface tension measurements. Equilibrium surface tension measurements were made by the Wilhelmy plate technique with a Krüss K12 tensiometer. The surface tensions equilibrate within minutes or several hours, depending on the surfactant concentration. Sets of measurements were taken until the change in surface tension was less than $0.08 \mathrm{mN} \mathrm{m}^{-1}$ in $15 \mathrm{~min}$.

PH measurements. $\mathrm{pH}$ was measured with a combination electrode (Thermo Orion, model $8102 \mathrm{BN}$ ) with a $2 \mathrm{M} \mathrm{KCl}$ filling solution saturated with $\mathrm{AgCl}$. The electrode was calibrated with Crison standard buffer solutions. Measurements were made at increasing concentration to minimize error due to electrode contamination.

\section{Results and discussion}

\section{Surface tension}

The surface tension as a function of concentration is shown in Fig. 2. A break can be observed at concentration $6 \times 10^{-2} \mathrm{mM}$ with a certain levelling of the surface tension but still decreasing to complete levelling off around $1 \times 10^{-1} \mathrm{mM}$. From the slope just before the first levelling at $6 \times 10^{-2} \mathrm{mM}$ the surface excess can be calculated according to the Gibbs adsorption isotherm in eqn. (8):

$$
\Gamma=-\frac{1}{n R T} \frac{\mathrm{d} \gamma}{\mathrm{d}(\ln c)}
$$

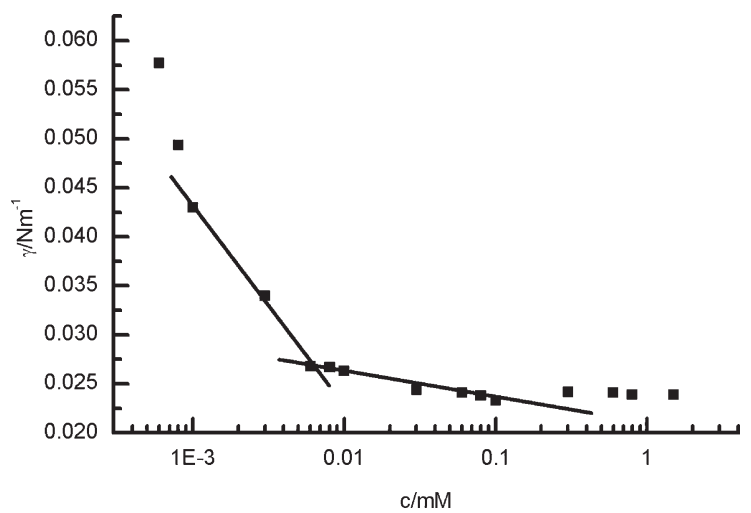

Fig. 2 Surface tension as a function of concentration of aqueous solutions of $1010 \mathrm{R}$ at $25^{\circ} \mathrm{C}$ obtained by the Wilhelmy plate method. where $\gamma$ is the surface tension, $c$ the bulk surfactant concentration, $R$ the gas constant, $T$ the absolute temperature. The parameter $n$ is the effective number of dissociated species per molecule for solutions without added salt. In the case of 1010R surfactant $n=3$; the cationic surfactant plus two chloride ions (see Fig. 1).

The experimental value of surface excess was $1.19 \times 10^{-6}$ mol m$~^{-2}$. From the surface excess $\Gamma$, the area per molecule is obtained via Avogadro's number, eqn. (9).

$$
A_{\mathrm{m}}=\frac{1}{\Gamma N_{\mathrm{A}}}
$$

The calculated area per surfactant molecule was $1.4 \mathrm{~nm}^{2}$. The consideration of smaller adsorbing species numbers would lead to smaller areas per molecule, i.e. $0.93 \mathrm{~nm}^{2}$ for $n=2$ and $0.47 \mathrm{~nm}^{2}$ for $n=1$.

The surface tension curve could be qualitatively described taking in consideration the ionisation of the surfactant and the concentration dependence on the relative concentration of species. See below for a more detailed discussion about the species in solution. At low surfactant concentration the dominant species would be the singly charged molecule, which, because of lower water solubility is expected to be more surface active than the doubly charged one. At high concentration the proportion of doubly charged species will dominate, producing a modest decrease of surface tension because of the adsorption of the additional chloride ion. The higher slope at very low concentration, if true, could be due to the adsorption of the non-charged species. However, the amount of this form in the acid-base equilibrium seems to be too low to justify this affirmation. The shallow minimum around $10^{-4} \mathrm{M}$ could be due to the desorption of the lower charged species by competition with the higher charged species and their solubilisation in micelles, much in the same way the dodecanol present in dodecylsulfate solutions induce a minimum in the surface tension around the critical micellar concentration.

\section{pH behaviour}

The $\mathrm{pH}$ as a function of surfactant concentration at $25^{\circ} \mathrm{C}$ is shown in Fig. 3. Because the surfactant molecule can dissociate in several species, the concentration behaviour is relatively complex. The species in solution and the different equilibrium are shown in Scheme 1. The salt dissociation is expected to be a complete reaction.

Without taking into consideration the possibility of preferential adsorption of certain species or the aggregation in the bulk solution and assuming dilute ideal behaviour, we can

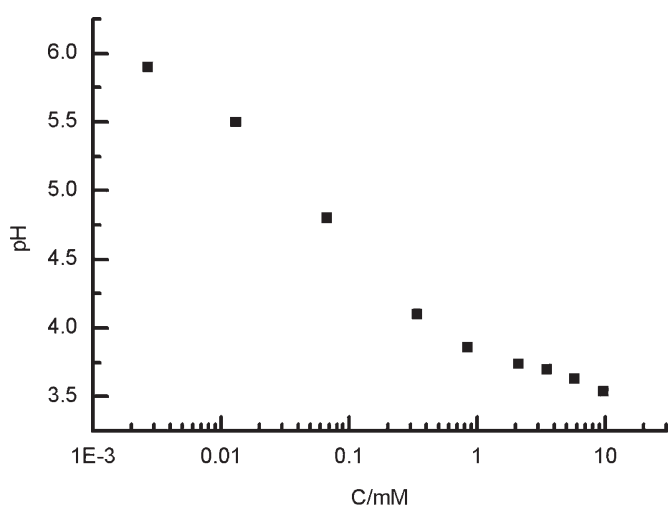

Fig. $3 \mathrm{pH}$ as a function of concentration of aqueous solutions of $1010 \mathrm{R}$ at $25^{\circ} \mathrm{C}$. 

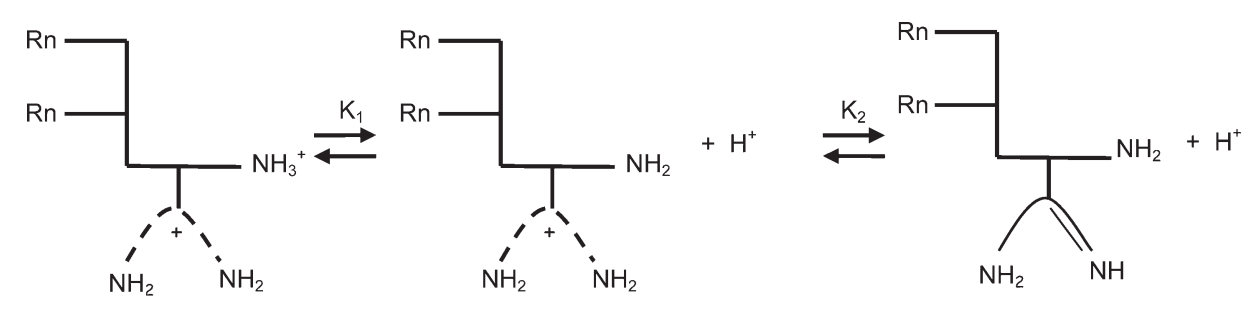

Scheme 1 Acid-base equilibrium of the 1010R surfactant in aqueous solution.

solve the acid-base equilibrium equations resulting in eqn. (10):

$$
K_{1}=\frac{\left[\mathrm{H}^{+}\right]-\frac{K_{\mathrm{w}}}{\left[\mathrm{H}^{+}\right]}}{C_{\mathrm{a}}\left(\frac{1}{\left[\mathrm{H}^{+}\right]}+\frac{2 K_{2}}{\left[\mathrm{H}^{+}\right]^{2}}\right)-\left(\frac{1}{\left[\mathrm{H}^{+}\right]}+\frac{K_{2}}{\left[\mathrm{H}^{+}\right]^{2}}\right)\left(\left[\mathrm{H}^{+}\right]-\frac{K_{\mathrm{w}}}{\left[\mathrm{H}^{+}\right]}\right)}
$$

where $C_{\mathrm{a}}$ is the total concentration of surfactant, $K_{1}, K_{2}$ are the first and second dissociation constants and $K_{\mathrm{w}}$ the ionic product of water.

It should be mentioned that the water used does not have neutral $\mathrm{pH}$; this acidity is usually attributed to $\mathrm{CO}_{2}$ solubilisation which makes the $\mathrm{pH}$ lower than 7 . If we take this additional equilibrium into account, the results are very close to those obtained from eqn. (3) except for the lowest concentration. The dissociation constant of the guanidine group in the literature are of the order of $10^{-12} \cdot{ }^{29}$ By assuming $K_{2}=10^{-12}$ we can calculate $K_{1}$. In Fig. 4 we show the calculated $K_{1}$. It is clear that the dissociation constant is much higher that it could be assumed regarding the literature, the surfactant behaves as a weak acid (comparable to acetic acid), not as a very weak acid as expected for an ammonium group (the dissociation constant for protonated ethylamine is around $\left.2.5 \times 10^{-11}\right)$. The variation of this dissociation constant with concentration could be due to the aggregation of the molecule (note the maximum apparent dissociation constant around $1 \mathrm{mM}$ ). For the surfactant to release more protons (higher dissociation constant) in the aggregated state would mean that the aggregation takes place preferably with the dissociated species (neutral species in the present case). We should also take into account how adsorption of the surfactant on the glass electrode could influence those results. On the one hand it is expected that adsorption at interfaces will be stronger for the lower charged molecules (the more dissociated ones with this surfactant). However, the positive charge on the surfactant could also imply the contrary effect, higher adsorption of the more charged species because of the usual free negative charges on glass surfaces. If the adsorption of neutral species is favoured, then the concentration of protons would be locally

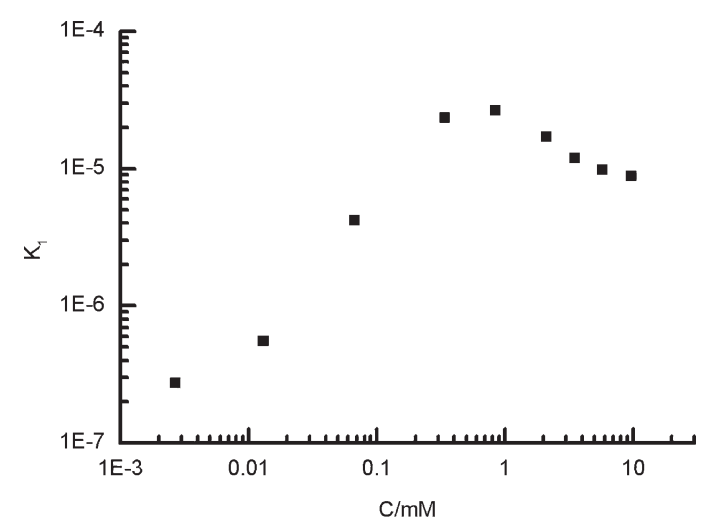

Fig. 4 Apparent first dissociation constant of the surfactant 1010R as a function of concentration. See the text for details. higher than in the bulk (see Scheme 1), explaining the relatively high dissociation constant found. If adsorption of the divalent cation was favoured then the concentration of protons would be lower than in the bulk, meaning that the apparent dissociation constant would be still higher than measured.

The higher than expected acid constant for this surfactant contrasts with the less acid than expected behaviour encountered in fatty acids. ${ }^{30}$ The reason for this difference should lie in the different charges on the fatty acids (the neutral molecule is the acid form) and in 1010R where the neutral form is the dibasic species. Kanicky et al. ${ }^{30}$ explain their results on the apparent $\mathrm{p} K$ of fatty acids with the argument that a monolayer with half the molecules ionised allow for the stabilisation of the acidic proton of one of the molecules in the pair. Following this argument with $1010 \mathrm{R}$ we should expect for the singly ionised and non ionised species to be favoured. This could explain the high acid constant. Letting $\mathrm{p} K_{1}$ and $\mathrm{p} K_{2}$ be adjustable parameters the best fit produces similar values for both constants (values around 5), however, the statistical error in their determination becomes quite high. Moreover, the $\mathrm{pH}$ concentration curve for the same molecule acetylated in the amino group seems to indicate that the apparent $\mathrm{p} K$ of the guanidine group is also of the order of $5 .{ }^{31}$

\section{Light scattering}

In Fig. 5 the scattered intensity at $90^{\circ}$ is plotted as a function of concentration for the surfactant $1010 \mathrm{R}$ at $25^{\circ} \mathrm{C}$. Two changes of slope are apparent in this figure. The first one, at a concentration of about $4 \times 10^{-2} \mathrm{mM}$ is close to the c.m.c. detected by surface tension while the second one, around $5 \times 10^{-1} \mathrm{mM}$, is close to the c.m.c. detected by conductivity and fluorescence. ${ }^{5}$ The closed symbols in Fig. 5 show the detail of the low concentration samples, note that for those points the intensity has been magnified by a factor of 100 . For reference, the level of our background water is shown as a horizontal line with the same scale as the lower concentrations.

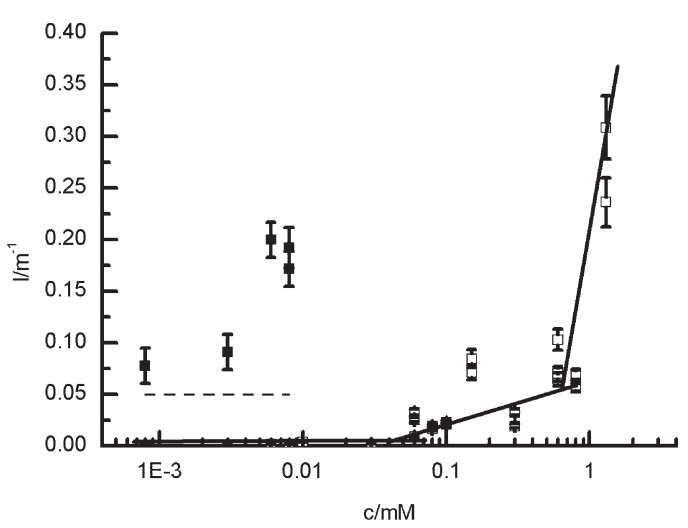

Fig. 5 Absolute scattered intensity at $90^{\circ}$ as a function of concentration of surfactant solutions at $25^{\circ} \mathrm{C}$. The closed points shows the detail of the low concentration scattered intensity, for those points the scale should be divided by 100 . The line corresponds to the water background scattering for comparison in the same scale as the closed points. 
Although the reproducibility of the intensity data is not very good at very low concentrations, it is clear that, even at those low concentrations some aggregation must be present, the intensity before background subtraction, is, at least, double than the background intensity. For some of the concentrations the intensity as a function of the scattering angle was recorded and this effect was more pronounced at lower scattering angles.

In Fig. 6 we show a selection of the angular dependence of the scattered light for different concentrations. The concentration of $8 \times 10^{-3} \mathrm{mM}$ (Fig. 6a) is lower than the c.m.c. as obtained by surface tension. The $6 \times 10^{-2} \mathrm{mM}$ (Fig. 6b) sample lies between the c.m.c. obtained by surface tension and that obtained by conductivity. The sample $6 \times 10^{-1} \mathrm{mM}$ (Fig. 6c) lies around the c.m.c. detected by conductivity and that with $1.3 \mathrm{mM}$ (Fig. 6d) is already above that value. In each plot three sets of points are shown, they correspond to the intensity, intensity multiplied by the scattering vector modulus $q$ and intensity multiplied by the squared scattering vector modulus as a function of the squared scattering vector modulus. Those correspond to Guinier plots for globular, rod and lamella respectively. Linear plots are obtained if the objects have the corresponding geometry. ${ }^{27}$ Although the scattering vector range is not large enough to directly prove the geometry, we can appreciate that changes are occurring at those concentrations. The triangles, which correspond to $I q^{2}$ (lamellar representation) have negative slope for $8 \times 10^{-3} \mathrm{mM}$ (Fig. 6a) and positive slope for $1.3 \mathrm{mM}$ (Fig. 6d). Positive slopes are incompatible with the structure detected in this range. Therefore while $8 \times 10^{-3} \mathrm{mM}$ could correspond to globular aggregates, $1.3 \mathrm{mM}$ can not correspond to lamellae. Moreover, the consideration of the apparent molecular weight calculated form the intensity at $45^{\circ}$ and the apparent gyration radii obtained from the Guinier plot, eqn. (5), using the angles of 45, 60 and $75^{\circ}$, shows that a model of compact spheres is inconsistent. The molecular weight calculated from the gyration radii is always more than one order of magnitude bigger than the experimental value. We should also note that performing these calculations the condition of validity of eqn. (5) is not strictly accomplished and $q R_{\mathrm{g}} \sim 2$ in the range of calculation. The structure that reconciles the overall intensity with its angular dependence is that of vesicles at low concentrations and ribbons at the higher concentration.

To further investigate this possible assignation we have tried to fit the form factor of vesicles and cylinders to the experimental data considering that the RDG approximation applies, ${ }^{28}$ together with the absence of interparticle interaction due to high dilution. We have calculated the form factors analytically

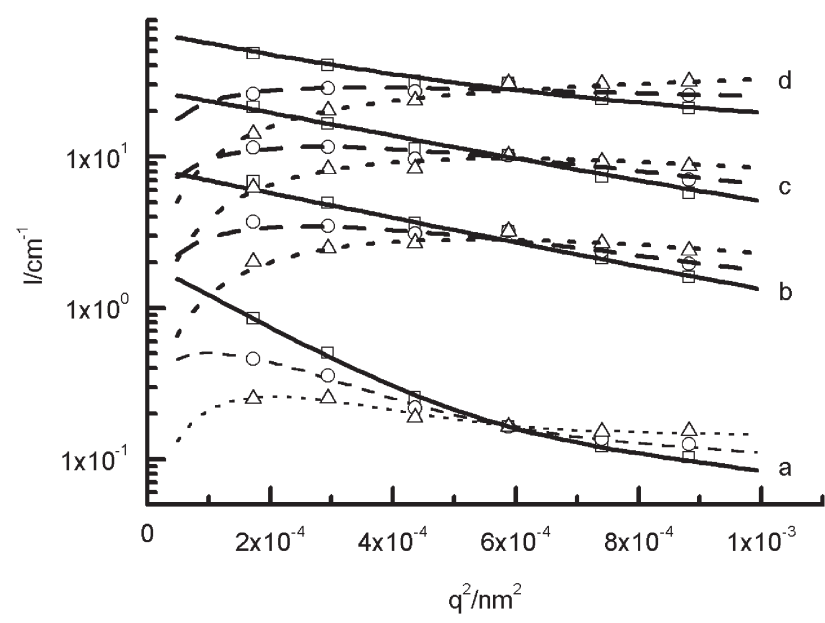

Fig. $6 I(\square), I q(\mathrm{O})$ and $I q^{2}(\bullet)$ as a function of $\mathrm{q}^{2}$. Model $I$ (full line), $I q$ (dashed lines), $I q^{2}$ (dotted lines), with increasing concentrations a, b, c, d. or by the Fourier transform of the pair distribution function of the corresponding object. ${ }^{24-25}$

We have further scaled the model to the absolute scattered scale to compare the goodness of the fit. The intensity and intensity multiplied by $q$ and $q^{2}$ are shown in Fig. 6 . The intensity trend as a function of angle is quite well represented by the form factor of the ellipsoidal vesicles or cylinders with elliptical section. Because the scattering vector range is relatively small, the dependence of the intensity trend with bilayer thickness is very weak, and this parameter mainly depends on the absolute level of intensity.

The fitting parameters for ellipsoidal vesicles are shown in Table $1 ; a, b$ and $c$ are the external axes of the ellipsoid and $d$ is the thickness of the bilayer. For the cylindrical model (1.3 $\mathrm{mM}$ and $5 \times 10^{-2} \mathrm{mM}$ at low $\left.\mathrm{pH}\right) a$ corresponds to the length of the cylinder; $b$ and $d$ are the semiaxes of the elliptical section of the cylinder. For those two last samples the elliptical vesicles could not fit the experimental values even with extreme parameters. The aggregation number, molecular weight and area per surfactant molecule have been calculated taking into account a surfactant molecular volume of $1.1 \mathrm{~nm}^{3}$. The calculated area per surfactant molecules seem excessive, not just compared to the values we obtain from surface tension but with the usual values found for double chain surfactants. ${ }^{32}$ We have to mention that the most concentrated sample can also be fitted by a flexible rod model with Gaussian statistics. In this case the fitted gyration radius corresponds to $78.6 \mathrm{~nm}$ and the molecular mass of the aggregate to $1.7 \times 10^{7}$, which is close to the value obtained from the elliptical section cylinder. It is clear from the data that the aggregation changes of this surfactant with concentration influences both the total mass of the aggregate and the form of the aggregate.

A slightly better quality of fit can be obtained by using a polydisperse population of vesicles with a Schultz distribu$\operatorname{tion}^{33}$ for the radii and constant thickness. The fitted parameters are shown in Table 2. $R$ and $\sigma$ are the mean and width of the Schultz distribution, $d$ is the bilayer thickness, $A_{\mathrm{m}}$ the area per molecule in the aggregates and $M_{\mathrm{w}}$ the aggregate molecular weight. From this data it is clear that the global size of the aggregates decreases as the concentration of surfactant increases, as the distribution width is nearly constant the relative polydispersity increases. The vesicle thickness and, the calculated area per molecule of surfactant have been scaled to reach the measured absolute intensity. The vesicular thickness decreases as the concentration increases and the area per molecule increases. An increase in area per molecule is what could be expected from the consideration of the charge per molecule. This model also fails for the most concentrated sample; small vesicles very polydispersed with large thickness and small area per molecule would be obtained. The quality of the fit in this case is also clearly worse as can be observed from the large uncertainty of the parameters.

As the anisometry of the elliptical section cylinders is very large, the same quality of fit can be obtained by using a ribbon model. The parameters of this fit are given in Table 3 for a 1.3 $\mathrm{mM}$ sample and for the $5 \times 10^{-2} \mathrm{mM}$ sample in presence of $\mathrm{HCl}$ with a final acid concentration of $0.04 \mathrm{M}$. The fit of both samples give reasonable agreement with the same area per surfactant molecule of $1.2 \mathrm{~nm}^{2}$ reinforcing the trend of increasing area per molecule as the surfactant concentration increases or with decreasing $\mathrm{pH}$.

The 1010R surfactant has a chiral carbon atom in the arginine residue (Fig. 1). In the literature some examples of ribbons formed by surfactants with a chiral carbon atom are described. ${ }^{34,35}$ The ribbons in those papers preferably adopt a helical structure. In the range of our experiment this helical structure has not any noticeable influence if the period of the helix is moderate.

In parallel, quasielastic light scattering was performed on the most concentrated samples. Although these results were 
Table 1 Fitted parameters of the ellipsoidal vesicles and elliptical section cylinders in the RDG approximation ${ }^{a}$

\begin{tabular}{|c|c|c|c|c|c|c|c|}
\hline Concentration/mM & $a / \mathrm{nm}$ & $b / \mathrm{nm}$ & $c / \mathrm{nm}$ & $d / \mathrm{nm}$ & $A_{\mathrm{m}} / \mathrm{nm}^{2}$ & $N_{\mathrm{a}}$ & $M_{\mathrm{w}} / \mathrm{g} \mathrm{mol}^{-1}$ \\
\hline $8 \times 10^{-3}$ & 175 & 155 & 35 & $0.90 \pm 0.1$ & $2.4 \pm 0.3$ & $2.7 \times 10^{5}$ & $1.7 \times 10^{8}$ \\
\hline $6 \times 10^{-2}$ & 120 & 70 & 35 & $1.32 \pm 0.2$ & $1.7 \pm 0.3$ & $1.1 \times 10^{5}$ & $6.5 \times 10^{7}$ \\
\hline $6 \times 10^{-1}$ & 100 & 100 & 20 & $0.47 \pm 0.1$ & $4.7 \pm 1$ & $9.5 \times 10^{4}$ & $6.0 \times 10^{7}$ \\
\hline 1.3 & 250 & 45 & & $0.86 \pm 0.2$ & $1.3 \pm 0.3$ & $3.2 \times 10^{4}$ & $2.0 \times 10^{7}$ \\
\hline $5 \times 10^{-2}$ & 100 & 100 & 35 & $1.06 \pm 0.1$ & $2.1 \pm 0.2$ & $1.5 \times 10^{5}$ & $7.3 \times 10^{7}$ \\
\hline $5 \times 10^{-2 b}$ & 320 & 50 & & $0.43 \pm 0.1$ & $2.6 \pm 0.6$ & $4.0 \times 10^{4}$ & $2.5 \times 10^{7}$ \\
\hline
\end{tabular}

${ }^{a} a, b$ and $c$ are the corresponding semiaxes for ellipsoidal vesicles, $d$ the vesicle bilayer thickness. For sample 1.3 and $5 \times 10^{-2}$ with $\mathrm{HCl} a$ is the cylinder length, $b$ and $d$ the semiaxes of the elliptical section. $A_{\mathrm{m}}$ is the area per molecule, $N_{\mathrm{a}}$ the aggregation number and $M_{\mathrm{w}}$ the molecular weight. b $5 \times 10^{-2} \mathrm{mM}$ with $\mathrm{HCl}$ to give a final concentration of $0.04 \mathrm{M}$.

Table 2 Fitted parameters for a Schultz distribution of spherical vesicles in the RDG approximation ${ }^{a}$

\begin{tabular}{lcccccc}
\hline Concentration $/ \mathrm{M}$ & $R / \mathrm{nm}$ & $\sigma / \mathrm{nm}$ & $\sigma / R$ & $d / \mathrm{nm}$ & $A_{\mathrm{m}} / \mathrm{nm}^{2}$ & $M_{\mathrm{w}} / \mathrm{g} \mathrm{mol}{ }^{-1}$ \\
\hline $8 \times 10^{-3}$ & $104 \pm 5$ & $34 \pm 5$ & 0.33 & $4.3 \pm 0.2$ & $0.49 \pm 0.03$ & $2.7 \times 10^{5}$ \\
$6 \times 10^{-2}$ & $97 \pm 10$ & $49 \pm 13$ & 0.51 & $4.1 \pm 0.5$ & $0.51 \pm 0.06$ & $1.7 \times 10^{8}$ \\
$6 \times 10^{-1}$ & $59 \pm 8$ & $28 \pm 13$ & 0.47 & $2.9 \pm 0.5$ & $0.74 \pm 0.07$ & $9.5 \times 10^{5}$ \\
1.3 & $34 \pm 25$ & $24 \pm 28$ & 0.71 & $6.1 \pm 2.0$ & $0.35 \pm 0.2$ & $3.2 \times 10^{4}$ \\
$5 \times 10^{-2}$ & $73 \pm 1$ & $29 \pm 3$ & 0.40 & $5.4 \pm 0.2$ & $0.40 \pm 0.03$ & $2.0 \times 10^{7}$ \\
$5 \times 10^{-2 b}$ & $29 \pm 27$ & $24 \pm 30$ & 0.83 & $3.1 \pm 1.5$ & $0.71 \pm 0.3$ & $7.3 \times 10^{7}$ \\
& & & & & $4.0 \times 10^{4}$ & $2.5 \times 10^{7}$
\end{tabular}

${ }^{a} R$ is the vesicle radius, $\sigma$ the distribution width, $d$ the bilayer thickness, $A_{\mathrm{m}}$ the area per surfactant molecule, $N_{\mathrm{a}}$ the aggregation number and $M_{\mathrm{w}}$ the molecular weight of the aggregates. ${ }^{b} 5 \times 10^{-2} \mathrm{mM}$ with $\mathrm{HCl}$ to give a final concentration of $0.04 \mathrm{M}$.

not obtained in ideal conditions (large aperture of the photomultiplier) they agree overall with these observations. When forcing the model to produce a single population, this was a very wide distribution centred around $99 \mathrm{~nm}$ and, when allowed to fit a bimodal distribution the peaks were centred at 59 and $205 \mathrm{~nm}$ respectively, agreeing with the dimensions of the ribbon model.

The transition from vesicular to cylindrical geometry could be caused by an increase in the area per surfactant molecule or a decrease of the hydrophobic tail length or a combination of both. Not much can be said about this point with the present data, only the trend is apparent. The concentration used in the calculations corresponds to the nominal concentration. We have to note that if the aggregation corresponds only to a part of the surfactant (as it occurs with classical surfactants for which the concentration of aggregates correspond to the nominal concentration minus the c.m.c.) thickness and area per molecule could be different. However, to keep a constant bilayer thickness we would need a concentration dependent micellar concentration. This is not unreasonable in view of the acid-base behaviour of those surfactants. The higher the concentration of surfactant, the higher the proportion of the more charged species and higher the critical micellar concentration would be. Moreover, this interpretation could reconcile de widely different values of critical micellar concentration depending on the method used for its determination. It has been found that the values of critical micellar concentration

Table 3 Fitted parameters for a ribbon model in the RDG aproximation $^{a}$

\begin{tabular}{|c|c|c|c|c|c|}
\hline $\begin{array}{l}\text { Concentration/ } \\
\mathrm{mM}\end{array}$ & $\begin{array}{l}L / \\
\mathrm{nm}\end{array}$ & $\begin{array}{l}W / \\
\mathrm{nm}\end{array}$ & $\begin{array}{l}d / \\
\mathrm{nm}\end{array}$ & $\begin{array}{l}A_{\mathrm{m}} / \\
\mathrm{nm}^{2}\end{array}$ & $\begin{array}{l}M_{\mathrm{w}} / \\
\mathrm{g} \mathrm{mol}^{-1}\end{array}$ \\
\hline $\begin{array}{l}1.3 \\
5 \times 10^{-2 b}\end{array}$ & $\begin{array}{l}250 \pm 10 \\
450 \pm 30\end{array}$ & $\begin{array}{l}60 \pm 1 \\
31 \pm 2\end{array}$ & $\begin{array}{c}1.90 \pm 0.05 \\
1.7 \pm 0.1\end{array}$ & $\begin{array}{l}1.2 \\
1.2\end{array}$ & $\begin{array}{l}2.0 \times 10^{7} \\
2.5 \times 10^{7}\end{array}$ \\
\hline \multicolumn{6}{|c|}{$\begin{array}{l}{ }^{a} L \text { is the length of the ribbon, } W \text { its width and } d \text { its thickness. } A_{\mathrm{m}} \text { the } \\
\text { area per surfactant molecule and } M_{\mathrm{w}} \text { the molecular weight. }{ }^{b} 5 \times 10^{-2} \\
\mathrm{mM} \text { with } 0.04 \mathrm{M} \text { of } \mathrm{HCl}\end{array}$} \\
\hline
\end{tabular}

are about one order of magnitude lower as obtained from surface tension measurements than that obtained from conductivity or fluorescence. Surface tension measurements will sense first the lower charged species; as the concentration increases the acid-base equilibrium favours the presence of more charged species, which will also adsorb and replace the lower charged species. Although, not very clear with the present data, this change could correspond to the very shallow minimum that is observed around $10^{-1} \mathrm{mM}$. The more charged species would then micellate. This explanation also agrees qualitatively with the observed intensity as a function of concentration. At very low concentration some non-negligible scattering occurs. This can be seen in Fig. 5, the closed symbols are a magnification of the open symbols and are compared with the level of the subtracted background, which has a magnitude a few times above the water background scattering. Between $10^{-3}$ and $10^{-1} \mathrm{mM}$ the intensity starts growing with concentration and from $1 \mathrm{mM}$ the intensity starts growing at a faster rate. In the very low concentration some aggregation of the non-charged species can be expected even at those very low concentrations. Increasing concentration the dominant species would be the singly charged species and at even higher concentrations the double charged species.

Additional proof of the effect of $\mathrm{pH}$ on the aggregation of this surfactant was obtained by adding a small amount of hydrochloric acid solution to a sample in which the preferred structure was vesicular. The changes observed are quite dramatic as it can be observed in Fig. 7. In this figure the scattered intensity as a function of dispersion modulus is plotted for a sample $5 \times 10^{-2} \mathrm{mM}$ and the same sample after addition of aqueous $\mathrm{HCl}$ solution with a final concentration of $0.04 \mathrm{M}$ $\mathrm{HCl}$. The intensity at $90^{\circ}$ dropped by a factor of four and the curvature of the plot also changed. This is more easily seen by comparing the $I q$ curve for both samples. Without acid addition this curve has a clear negative slope in most of the range while the acidified sample presents a flat $I q$ behaviour. This change can be compared to the change observed between Fig. $6 \mathrm{~b}$ and Fig. $6 \mathrm{~d}$.

The curves in Fig. 7 correspond to polydisperse vesicles for the original sample and a ribbon model for the acidified 


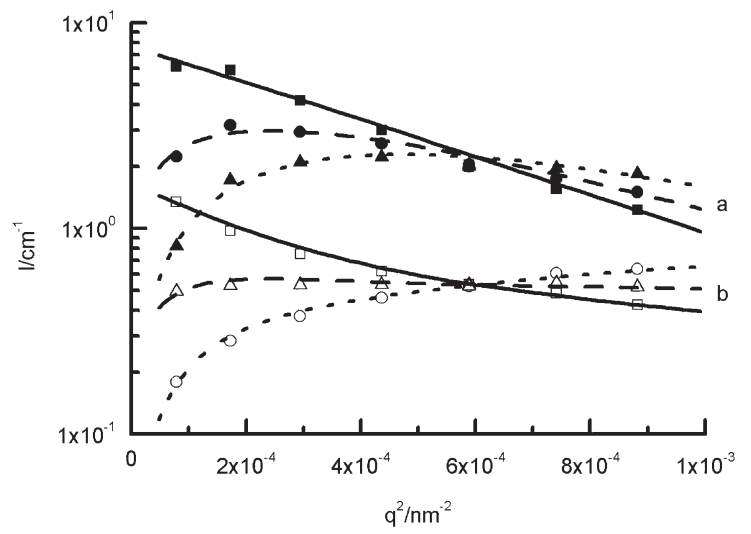

Fig. $7 I(\square), I q(\bigcirc)$ and $I q^{2}(\bullet)$ as functions of $q^{2}$. Model $I$ (full line), $I q$ (dashed lines), $I q^{2}$ (dotted lines). Closed symbols correspond to $5 \times 10^{-5} \mathrm{M}$ and open symbols to this sample after acidification to $0.04 \mathrm{M} \mathrm{HCl}$.

sample. The parameters of the fits are also shown in Tables 1,2 and 3. Those parameters compare close to the sample of $6 \times 10^{-2} \mathrm{mM}$ and $1.3 \mathrm{mM}$ respectively, with assigned vesicular and ribbon structures respectively. This shows that increasing concentration and decreasing $\mathrm{pH}$ have a similar effect on the aggregates and further strengthen the conclusion that the surfactant concentration influences the structure via a $\mathrm{pH}$ effect.

\section{Conclusions}

The aggregation properties of 1010R surfactant have been measured by means of static light scattering, surface tension and $\mathrm{pH}$ in the very diluted region. The apparent molecular weights of the aggregates are very high and tend to decrease as the concentration increases. Increasing the concentration also decreases the slope of the intensity as a function of scattering angle. The results allow for the assignation of a vesicle to ribbon transition to this process. The rational behind this transition corresponds to an increase of the area per surfactant molecule as the concentration increases induced by $\mathrm{pH}$ reduction as the surfactant concentration increases. Confirmation of this extreme was achieved with a vesicular sample which shows transitions to ribbon structure on decreasing $\mathrm{pH}$.

\section{Acknowledgements}

We are grateful to I. Carrera for technical assistance in the LS experiments and to Spanish CICYT for partial support of this work through Grants MAT2001-1188-CO2-02 and PPQ20001687-CO2-01.

\section{References}

1 M. R. Infante, J. Garcia Dominguez, P. Erra, R. Julia and M. Prats, Int. J. Cosmet. Sci., 1984, 6, 275-282.

2 L. Perez, J. L. Torres, A. Manresa, C. Solans and M. R. Infante, Langmuir, 1996, 12, 5296-5301.
3 A. Pinazo, X. Wen, L. Pérez, M. R. Infante and E. I. Franses, Langmuir, 1999, 15, 3134-3142.

4 L. Pérez, A. Pinazo, P. Vinardell, M. Angelet, P. Clapés and M. R. Infante, New J. Chem., 2002, 26, 1221-1227.

5 L. Pérez, M. R. Infante, M. Angelet, P. Clapés and A. Pinazo, Prog. Colloid Surf., 2003 (DOI: 10.1007/b11628).

6 P. C. Hiemenz and R. Rajagopalan, in Principles of Colloid and Surface Chemistry, Marcel Dekker, New York, ch. 8, p. 371

7 C. Tanford, The Hydrophobic Effect, Wiley, New York, 1973, 1980.

8 C. Tanford, J. Phys. Chem., 1972, 76, 3020-3024.

9 J. N. Israelachvili, D. J. Mitchell and B. W. Ninham, J. Chem. Soc., Faraday Trans. 1, 1976, 72, 1525-1568.

10 J. N. Israelachvili, D. J. Mitchell and B. W. Ninham, Biochim. Biophys. Acta, 1977, 470, 185-201.

11 V. K. Aswal, S. De, P. S. Goyal, S. Bhattacharya and R. K. Heenan, J. Chem. Soc., Faraday Trans., 1998, 94, 2965-2967.

12 S. U. Egelhaaf and P. Schurtemberger, J. Phys. Chem., 1994, 98, $8560-8573$.

13 C. Treiner, A. K. Chattopadhyay and R. Bury, J. Colloid Interface Sci., 1985, 104, 569-578.

14 F. Quiron and J. E. Desnoyers, J. Colloid Interface Sci., 1986, 112, $565-572$.

15 S. Ikeda and K. Fujio, Colloid Polym. Sci., 1992, 270, 1009-1017.

16 A. Stradner, O. Glatter and P. Schurtenberger, Langmuir, 2000, 16, 5354-5364.

17 R. Zana, J. Colloid Interface Sci., 2002, 248, 203-220.

18 R. Zana, Adv. Colloid Interface Sci., 2002, 97, 205-253.

19 R. Zana, in Structure-Performance Relationships in Surfactants, ed. K. Esumi and M. Ueno, Marcel Dekker, New York, 2003, ch. 6 , pp. 255-283.

20 A. Pinazo, X. Wen, Y. Ch. Liao, A. Prosser and E. I. Franses, Langmuir, 2002, 18, 8888-8896.

21 A. Walter, G. Kuehl, K. Barnes and G. VanderWaerdt, Biochim. Biophys. Acta, 2000, 1508, 20-33.

22 S. U. Egelhoaf and P. Schurtemberger, J. Phys. Chem., 1994, 98, $8560-8573$

23 M. Hofer, in Neutron, X-Ray and Light Scattering: Introduction to an Investigative Tool for Colloidal and Polymeric Systems, ed. P. Lindner and Th. Zemb, North-Holland Delta Series, Amsterdam, part VI, pp. 301-324.

24 O. Glatter, in Neutron, X-Ray and Light Scattering: Introduction to an Investigative Tool for Colloidal and Polymeric Systems, ed. P. Lindner and Th. Zemb, North-Holland Delta Series, Amsterdam, part I, pp. 33-82.

25 J. S. Pedersen, Adv. Colloid Interface Sci., 1997, 70, 171-210.

26 O. Glatter and M. Hofer, J. Colloid Interface Sci., 1988, 122, $484-495$.

27 J. P. Cotton, in Neutron, X-Ray and Light Scattering: Introduction to an Investigative Tool for Colloidal and Polymeric Systems, ed. P. Lindner and Th. Zemb, North-Holland Delta Series, Amsterdam, part I, pp. 3-31.

28 J. M. Van Zanten and H. G. Monbouquette, J. Colloid Interface Sci., 1994, 165, 512-518.

29 B. Rzeszotarska and E. Masiukiewicz, Org. Prep. Proc. Int., 1988, 20, 427-464.

30 J. R. Kanicky and O. Shah, Langmuir, 2003, 19, 2034-2038.

31 L. Pérez, A. Pinazo, R. Pons, M. Angelet, N. Azemar and M. R. Infante, in Abstracts of 14th Surfactant In Solution Symposium, Universitat de Barcelona, 2002, p. 178, http://www.ub.es/ sis2002/SIS $\% 2025$.pdf

32 M. Rosen, Surfactants and Interfacial Phenomena, John Wiley and Sons, New York, 2nd edn., 1989.

33 L. H. Hanus and H. J. Ploehn, Langmuir, 1999, 13, 3091-3100.

34 N. Sommerdijk, M. Lambermon, M. Feiters, R. Nolte and B. Zwanenburg, Chem. Commun., 1997, 1423-1424.

35 R. Oda, I. Huc, M. Schmutz, S. J. Candau and F. C. Mackintosh, Nature, 1999, 399, 566-569. 